\title{
Degeneration of the suprachiasmatic nucleus in an Alzheimer's disease mouse model monitored by in vivo magnetic resonance relaxation measurements and immunohistochemistry
}

Upasana Roy a,b, Mónica T. Heredia-Muñoz ${ }^{\mathrm{a}}$, Lara Stute ${ }^{\mathrm{a}, \mathrm{c}}$, Corinna Höfling ${ }^{\mathrm{c}}$, Jörg Matysik ${ }^{\mathrm{b}}$, Johanna H. Meijer ${ }^{\mathrm{d}}$, Steffen Roßner ${ }^{\mathrm{c}}$, A. Alia ${ }^{\mathrm{a}, \mathrm{e}^{*}}$

anstitute of Medical Physics and Biophysics, University of Leipzig, Härtelstraße 16-18, D04107 Leipzig, Germany.

${ }^{b}$ Institute of Analytical Chemistry, University of Leipzig, Linnéstraße 3, D-04103

Leipzig, Germany.

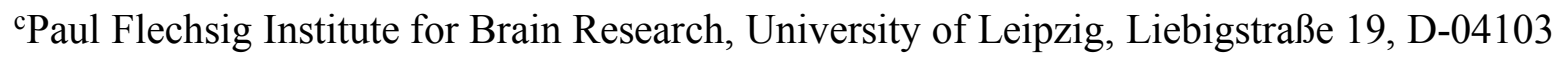
Leipzig, Germany.

${ }^{\mathrm{d}}$ Laboratory for Neurophysiology, Department of Cell and Chemical Biology, Leiden University Medical Center, Leiden, The Netherlands

${ }^{\mathrm{e}}$ Leiden Institute of Chemistry, Leiden University, Einsteinweg 55, 2333 CC Leiden, The Netherlands.

Running title: Disruption of suprachiasmatic nucleus in $\mathrm{AD}$

*Corresponding author: Institute of Medical Physics and Biophysics, University of Leipzig, Härtelstraße 16-18, D-04107 Leipzig, Germany; Tel.: +49 34197 15707; E-mail addresses: alia.aliamatysik@medizin.uni-leipzig.de; a.alia@.chem.leidenuniv.nl 


\section{Abstract}

In Alzheimer's disease (AD), disturbances in the circadian rhythm and sleep-wake cycle are frequently observed. Both are controlled by the master clock: the suprachiasmatic nucleus ( $\mathrm{SCN})$, which was reported in post mortem studies of $\mathrm{AD}$ subjects to be compromised. However, the influence of age and gender on the biophysical integrity and subtle microstructural changes of SCN and mechanistic connections between SCN dysfunction and AD progression in vivo remain to be explored. In the present study, we utilized state-of-the-art in vivo magnetic resonance relaxation measurements in combination with immunohistochemistry to follow microstructural changes in SCN of the Tg2576 (TG) mouse model of $\mathrm{AD}$. Longitudinal monitoring of in vivo $T_{2}$ relaxation with age shows significant shortening of $T_{2}$ values in the SCN of TG mice and more substantially in female TG than aged-matched controls (WT). Multiexponential $T_{2}$ analysis detected a unique long $T_{2}$ component in SCN of TG mice which was absent in WT mice. Immunohistochemical examination revealed significantly elevated numbers of activated astrocytes and an increase in the astrocyte to neuron ratio in SCN of TG compared to WT mice. This increase was more substantial in female than in male TG mice. In addition, low GABA production in SCN of TG mice was detected. Our results offer a brief appraisal of SCN dysfunction in AD and demonstrate that inflammatory responses may be an underlying perpetrator for the changes in circadian rhythmicity and sleep disturbance in $\mathrm{AD}$ and could also be at the root of marked sex disparities observed in $\mathrm{AD}$ subjects.

Keywords: Alzheimer's disease, gender difference, suprachiasmatic nucleus, $T_{2}$ relaxation time, $\operatorname{Tg} 2576$ mouse model 


\section{INTRODUCTION}

The suprachiasmatic nucleus ( $\mathrm{SCN}$ ) is assigned as the master clock in mammals, which enables anticipation and adaptation to the day night cycle. Individual neurons of the SCN are capable to generate circadian rhythms in a cell autonomous manner, displaying cyclic expression of clock genes and proteins $[1,2]$. The later lead to a rhythm in membrane excitability, in electrical activity and in a rhythmic release of humoral signals resulting in several rhythmic physiological processes [2]. The neurotransmitter $\gamma$-aminobutyric acid (GABA) is important for synchronization of tissue-level rhythms and intercellular signalling in the SCN [3-5], and is the only neurotransmitter that is produced and received by nearly all SCN neurons $[6,7]$. GABA is densely distributed in the $\mathrm{SCN}$ and located in the majority of neuronal somata and synaptic terminals [8]. Nevertheless, the role of GABA in the SCN is still not well understood.

The disruption of circadian rhythms and sleep wake cycle are common symptoms in Alzheimer's disease (AD) and senescence [9-12]. Disturbances in circadian rhythms of human $\mathrm{AD}$ patients [13] and in several $\mathrm{AD}$ mouse models [14, 15] have been reported. The evidences for SCN abnormalities seen in AD mainly derived from post mortem studies. These studies reported an alteration in the neuronal structure and function of the SCN during senescence and $\mathrm{AD}$ [13-15]. The changes in biophysical integrity and subtle microstructural changes in SCN during $\mathrm{AD}$ in vivo and its role in disease onset and progression remain unclear. Emerging evidence suggests that females are at greater risk of developing AD dementia [16-18], but the molecular mechanism accounting for sex-related differences in AD remains unknown. A morphometric study of SCN in the human brain revealed that the shape of the SCN in healthy subjects is sexually dimorphic, although SCN volume and cell number were equal in men and women [11]. A decline in SCN volume and cell number occurs during senescence as well as in $\mathrm{AD}$ patients [12]. Vasoactive intestinal polypeptide (VIP) cell 
number, in particular, decreases more significantly in pre-senile $\mathrm{AD}$ female patients than male patients [12]. Thus, the SCN shows gender differences in healthy subjects and AD patients. Up to now, no in vivo studies so far addressed whether and how sex differences in SCN is related with progression of $\mathrm{AD}$ pathology.

The quantitative transverse relaxation time $\left(T_{2}\right)$ measurement has proven to be a powerful non-invasive magnetic resonance imaging (MRI) tool which provides specific information about changes in tissue microstructure such as demyelination, axonal injury, gliosis and iron deposition [19-22]. and can be used as a marker for the assessments of normal aging and a variety of brain diseases including $\mathrm{AD}$ [23-25]. In a long-term follow up traumatic brain injury study, a clear relationship was established between $T_{2}$ relaxation time and the power spectrum of the electroencephalogram (EEG) [26]. Furthermore, multicomponent $T_{2}$ relaxation analysis is very sensitive to distinguish between various tissue water compartments and to distinguish the processes of inflammation and myelin loss [27]. Thus, monitoring in vivo $T_{2}$ changes in SCN during $\mathrm{AD}$ progression and examining changes in multicomponent $T_{2}$ relaxation may provide evidence for dynamic microscopic changes in the SCN, yielding new insights into the biophysical complexity of the underlying circadian rhythm disturbances in AD. Studies using transgenic mouse models of $\mathrm{AD}$ have the potential to clarify these in vivo changes in SCN. Tg2576 is a widely used model of AD, which develops $\mathrm{A} \beta$ plaques (one of the main hallmarks of AD) and it shows a progressive learning and memory impairment [28]. Previous studies employing electroencephalography (EEG) have reported changes in the circadian rhythms and sleep abnormalities of the Tg2576 AD mouse model [29].

In this study, in vivo $T_{2}$ analysis along with immunohistochemistry has been applied to identify and characterize age and gender-specific changes in SCN during progression of AD pathology in the Tg2576 mouse model. Our results show that inflammatory responses 
disturbed the biophysical integrity of SCN in Tg2576 mouse brain and a disruption of the SCN may be accountable for the gender-specific differences in the circadian rhythmicity that have been observed in $\mathrm{AD}$ patients.

\section{MATERIALS AND METHODS}

\section{Mouse model}

The transgenic $\operatorname{Tg} 2576$ mice used in this study express as transgene the human amyloid precursor protein (APP695) carrying the Swedish double mutation [28]. The transgene is expressed in C57B6/SJL breeders. The N2 generation mice of both genders (10 male and 10 female) were studied at ages between 10 and 18 months. Age-matched non-transgenic littermates (10 male and 10 female) served as controls. All animal experiments were approved by the Landesdirektion Sachsen (license T28/16) and UDEC commission Leiden University (license number 14199). All methods were carried out in accordance with the relevant guidelines and regulations.

\section{Brain Preparation and Histology}

Following in vivo MR measurements, mice were sacrificed by $\mathrm{CO}_{2}$ inhalation and transcardially perfused with phosphate-buffered saline (PBS, $\mathrm{pH}$ 7.4) followed by $4 \%$ buffered paraformaldehyde (Zinc Formal-Fixx, ThermoShandon, UK) through the left cardiac ventricle. After perfusion fixation the brain was dissected out and placed in the same fixative for 48 h. Following fixation, the brain was dehydrated and embedded in paraffin. Subsequently coronal sections (30 $\mu \mathrm{m}$ thick) were carefully cut using a vibratome while maintaining as much as possible the same spatial orientation of mouse brain as in the MR imaging experiments as described previously [30]. To stain activated astrocytes, brain sections were subjected to immunohistochemistry using a primary polyclonal anti-GFAP 
(glial fibrillary acidic protein) antibody (DAKO) as described earlier [17]. Immunolabeling was visualized by using the $\mathrm{ABC}$ kit (Vectastain) according to the manufacturer's instructions. Images of the histological sections were obtained using a Leica DM RE HC microscope, interfaced to a Leica DC500 3CCD digital camera.

For preparing sections for fluorescent labelling, after perfusion brains were dissected and placed in the same fixative for $24 \mathrm{~h}$ at $4^{\circ} \mathrm{C}$. After cryoprotection in $30 \%$ sucrose in $0.1 \mathrm{M}$ phosphate buffer for 3 days, coronal sections $(30 \mu \mathrm{m})$ were cut on a sliding microtome and collected in $0.1 \mathrm{M}$ phosphate buffer containing $0.025 \%$ sodium azide. For fluorescent labelling of $\mathrm{A} \beta$ deposition using thioflavin-S (ThS), sections were incubated with $1 \% \mathrm{ThS}$ (Sigma) for $20 \mathrm{~min}$, followed by $40 \mathrm{~min}$ incubation in $80 \%$ ethanol and a final rinse in distilled water, dehydrated in toluene and covered with coverslip using Entellan. For fluorescent immunolabeling, mouse brain slices were pretreated with $60 \%$ methanol (30 min), followed by washes in $0.1 \mathrm{M}$ TBS and blocked in TBS containing $0.3 \%$ TitonX-100 and 5\% goat serum for $30 \mathrm{~min}$. Subsequently slices were incubated overnight with a Cy3-conjugated mouse anti-GFAP antibody (for labelling of astrocytes) $(1: 1,000)$, mouse-anti-NeuN (for labelling of neuronal nuclei) (clone A60; 1:100; MAB 377, Millipore, Billerica, MA, USA) and rabbit anti-GAD antibody (for GABAergic neurons) (1:4,000). After washing in PBS three times, sections were incubation with corresponding fluorescence secondary antibodies (Dianova 1:400) for $1 \mathrm{~h}$.

Confocal laser scanning microscopy (LSM 510, Zeiss, Oberkochen, Germany) was performed to reveal co-localization of GFAP, NeuN, GAD and ThS staining. ThS (green fluorescence) was visualized by excitation with an argon laser at $488 \mathrm{~nm}$ and detection of emission at 510 $\mathrm{nm}$ using a low-range band pass $(505-530 \mathrm{~nm})$. For Cy3-labelled antigens (red fluorescence), a helium-neon laser with $543 \mathrm{~nm}$ excitation was used and emission from Cy3 at $570 \mathrm{~nm}$ was 
detected applying high-range band pass $(560-615 \mathrm{~nm})$. The Cy5-labelled antigens were visualized using excitation at $650 \mathrm{~nm}$ and emission at $670 \mathrm{~nm}$. Specificity of primary antibody and immunoreaction was confirmed by omitting primary antibodies or changing probes of the secondary antibodies.

\section{MRI experiments}

All MRI measurements were performed using $750 \mathrm{MHz}(17.6 \mathrm{~T})$ vertical $89-\mathrm{mm}$ bore magnets equipped with a $1 \mathrm{Tm}^{-1}$ actively shielded imaging gradient insert (Bruker, Germany). A birdcage radio-frequency (RF) coil with an inner diameter $2 \mathrm{~cm}$ was used for excitation and detection. The system was interfaced to a Linux PC running Topspin 2.0 and ParaVision 5.0 software (Bruker Biospin GmbH, Germany).

For in vivo $\mu \mathrm{MRI}$ measurements the mice were anesthetized with $2 \%$ isoflurane (Forane, Abbott, UK) in air $(0.3 \mathrm{~L} / \mathrm{min})$ and oxygen $(0.3 \mathrm{~L} / \mathrm{min})$. During scanning the level of anesthetic was maintained between 1 and $1.5 \%$ to keep the breathing of the animal at a constant rate of $\sim 50$ breaths per minute and the respiration rate was constantly monitored using a respiration sensor, (placed on the animal's abdomen) connected to a respiration unit. The respiration unit was connected to a computer with Bio-SAM respiration monitoring software (Bruker Biospin, Germany). The mouse's body temperature was kept at a constant temperature by pumping warm water through the gradient system. Cooling of the gradient was performed by circulating water.

$T_{2}$ relaxation time were measured with the Multi-Slice, Multi-Echo (MSME) sequence which is based on the Carr-PurcellMeiboom-Gill (CPMG) sequence, where transverse magnetization of a $90^{\circ}$ pulse is refocused by a train of $180^{\circ}$ pulses generating a series of echoes $[31,32]$. The following imaging parameters were used: Number of averages $(\mathrm{NA})=2$; Number of slices $(\mathrm{NS})=10 ;$ Number of echoes $=12$ with echo spacing $=6.064 ;$ A repetition time $(\mathrm{TR})=2 \mathrm{~s}$ 
with an effective spectral bandwidth $=100 \mathrm{kHz}$; Field of view $(\mathrm{FOV})=2.0 \times 2.0 \mathrm{~cm}$; Matrix size $=256 \times 256$; This yields an effective in-plane resolution of $0.078 \times 0.078 \mathrm{~mm}$ and a voxel resolution of $6.10 \times 10^{-3} \mathrm{~mm}^{3}$. The total acquisition time for the experiment was $17 \mathrm{~min}$ and 2 s. To study the dependence of $T_{2}$ on the CPMG refocusing interpulse interval $(\tau)$, the $T_{2}$ measurements were performed using the MSME sequence with 16 echo and 4 different refocusing interpulse intervals, namely 5.6, 8.5, 10, and $18 \mathrm{~ms}$. The last 5 echo of $8.5 \mathrm{~ms}$ acquisition, the last 7 echo of $10 \mathrm{~ms}$ acquisition and the last 10 echo of $18 \mathrm{~ms}$ acquisition were discarded to provide comparable temporal sampling windows (namely, 5.6-91.04 ms, 8.593.50 ms, 10-90 ms, 18-90 ms acquisitions, respectively). For the precise localization of regions of interest (ROIs) on the brain regions for $T_{2}$ measurements, a pilot scan of a mouse brain was acquired with multi-slice rapid acquisition using the relaxation enhancement (RARE) sequence [33] and subsequently ROIs were transferred to $T_{2}$ maps to ensure precise regional placement. The following imaging parameters were used for the RARE sequence: Echo time $(\mathrm{TE})=8.5 \mathrm{~ms} ; \mathrm{TR}=2000 \mathrm{~ms} ; \mathrm{NA}=1$, rare factor $=6 ; \mathrm{NS}=10$, with slice thickness $0.5 \mathrm{~mm}$. To establish the test-retest reliability of $T_{2}$ measurements and to check systematic errors, the same C57BL6/SJL mice $(n=5)$ were scanned twice on two subsequent days.

\section{Data Processing}

\section{Estimation of $T_{2}$}

For the estimation of $T_{2}$ values, selected regions of interest (ROIs) were manually drawn on the images by using an image sequence analysis (ISA) tool package (Paravision 5, Bruker). Monoexponential fitting was then used to calculate $T_{2}$ using a monoexponential fit function $\left[\mathrm{y}=\mathrm{A}+\mathrm{C}^{*} \exp \left(-\mathrm{t} / T_{2}\right)\right]$, where $\mathrm{A}=$ Absolute bias, $\mathrm{C}=$ signal intensity, $T_{2}=$ transverse relaxation time $[22,34]$. ROIs were defined for the SCN, hippocampus, cingulate cortex and piriform cortex on a $T_{2}$-weighted image using the "Allen Brain Atlas" with the brain explorer 
program (http://mouse.brain-map.org) as the reference atlas. For all animals, the $T_{2}$ was the mean of the ROIs drawn on the right and left sides of the brain.

\section{Nonnegative least-squares (NNLS) algorithm for $T_{2}$ component analysis}

The $T_{2}$ decay curve was also fitted to a multicomponent $T_{2}$ model. Decay curves were decomposed into an arbitrary number of exponential components using the nonnegative leastsquares (NNLS) algorithm. The NNLS procedures were implemented using the MATLAB codes (MathWorks, Natick, MA) and carried out to analyze the optimum solution of the data from imaging experiments using the "lsqnonlin" function. The signal intensities as a function of the echo time in MRI methods can be written as:

$$
y\left(t_{i}\right)=\sum_{j=1}^{M} S_{j} e^{-t_{i} / T_{2 j}}+C, \quad i=1,2, \ldots, N
$$

where $N$ is the number of echoes, $t_{i}$ is the $i^{\text {th }}$ echo time, $y\left(t_{i}\right)$ is the signal intensity of the $i^{\text {th }}$ echo, $M$ is the number of $T_{2}$ components, $S_{j}$ is the intensity of the $j^{\text {th }} T_{2}$ component, and $C$ is a constant accounting for any offset of the signal. For both simulation and experimental data, 12 $(M=12) \mathrm{T}_{2 \mathrm{j}}$ values were used. One additional $T_{2}$ value, which was set to infinity, was added to simulate the constant item in the equation above. A "least-square based constraint" rule was used to obtain a $T_{2}$ distribution. The value of the regularization in the calculation was chosen in such a way that the estimated distribution of $T_{2}$ is similar to that of the known $T_{2}$ for experimentally calculated data. For each $T_{2}$ spectrum from the NNLS calculation, any $T_{2}$ component with an intensity below a specific threshold (peak area less than $0.1 \%$ of the total area) or with the $T_{2}$ value at the peak position lower or higher than a threshold $(0.5 \mathrm{~ms}$ and $300 \mathrm{~ms}$, respectively) was ignored. The NNLS procedure used in this work means that the 
results were acquired without a priori assumptions about the number of $T_{2}$ components and any initial guess of the solution.

\section{Image analysis of brain sections}

For quantitative analysis of histological images, the brain regions $(\mathrm{SCN}$, cingulate cortex (CG), piriforn cortex (PIR) and hippocampus (HC)) were outlined according to the "Allen Brain Atlas" with the brain explorer program (http://mouse.brain-map.org) as the reference atlas from histological images using PhotoShop. Subsequently, the delineated brain regions were exported and analyzed in Image J software (ImageJ, USA). By using the plug-in color deconvolution, the colors were unmixed and the stained area was selected, and subsequently, the number of particles were calculated by using a plug-in called Image-based Tool for Counting Nuclei (ITCN). The percentage of the total $A \beta$ area was calculated using analyze particles. The data were exported to Origin Pro v. 8 software for further analysis. For image co-registration, immunohistological images were matched with each other using common anatomical landmarks such as the ventricles, corpus callosum and hippocampal fissure using PhotoShop 7.0 (Adobe Systems, San Jose, CA).

\section{Statistics}

All statistical analyses (t-test and analysis of variance) of the MRI and histological quantifications were performed with Origin Pro v.8. The paired and/or unpaired Student's ttest was used to compare mean values. Statistical significance was assigned for values of $\mathrm{P}<$ 0.05. Kolmogorov-Smirnov normality tests were performed which confirmed that the longitudinal $T_{2}$ relaxation data were normally distributed for a particular age and genotype with a probability ranging from 0.5 to 0.9 . A Kruskal-Wallis one-way analysis of variance along with rank test was implemented on the MR data to compare the median of $T_{2}$ values for all group comparisons. This method confirms that samples come from the same population for 
a particular age, sex, and genotype. Tests for homogeneity of variances between different ages $(10,12,15$ and 18 month) were also computed. No statistically significant change in variance was observed in particular age for any genotype. Subsequent Mann-Whitney tests were used to make pairwise comparisons between different mice groups. To assess the reliability of measurements, the intra-class correlation coefficient $\left(\mathrm{ICC}_{2,1}\right)$ was computed using a two-way random effects analysis of variance (ANOVA) model and the absolute agreement definition $[35,36]$. An ICC close to 1.0 indicates high reliability. The ICC can only be 1.0 if the measures are identical.

\section{RESULTS}

Prior to the longitudinal $T_{2}$ study, the test-retest reliability of in vivo $T_{2}$ measurements was analyzed by imaging the same mice with an interval of 5 days. Fig. 1A shows the ROIs used to quantify $T_{2}$ relaxation time changes in $\mathrm{SCN}$ and three other brain regions (cingulate cortex, piriform cortex and hippocampus). Fig. 1B shows test-retest reliability results of in vivo $T_{2}$ measurements in these brain regions of wild-type mice. Reliability was found to be very high for the $\operatorname{SCN}(\mathrm{ICC}=0.73, \mathrm{P}<0.05)$ as well as for cingulate cortex $(\mathrm{ICC}=0.75, \mathrm{P}<0.05)$, piriform cortex $(\mathrm{ICC}=0.72, \mathrm{P}<0.05)$ and hippocampus $(\mathrm{ICC}=0.66, \mathrm{P}<0.05)$. No systematic error, as checked by a paired t-test $(\mathrm{P}>0.05, \mathrm{n}=5)$ was observed between the measurements. The effect of CPMG refocusing interpulse interval $(\tau)$ on $T_{2}$ for the SCN and hippocampus regions was examined in order to rule out any influence of magnetic field disturbances on $T_{2}$ changes. As shown in Fig. 1C, no statistically significant effect of the interpulse interval in the range of interest $(6.6$ and $12 \mathrm{~ms})$ was observed on the $T_{2}$ of the SCN as well as of the hippocampus. This data confirms that the changes observed in $T_{2}$ values in these brain regions depend on variations in tissue properties rather than magnetic field disturbances. 
The $T_{2}$ values of SCN and other brain regions of male and female Tg2576 and wild-type mice are given in Figure 2. Clearly, the $T_{2}$ values of the hippocampus, cingulate and piriform cortex of the 18 month old Tg2576 mice showed a significant decrease as compared to the agematched wild-type mice. The decline in hippocampal $T_{2}$ relaxation time was more significant in female as compared to male $\operatorname{Tg} 2576$ mice $[23,30,37]$. In the thalamus region, $T_{2}$ values did not show any significant change between wild-type and Tg2576 mice. In the SCN region, the $T_{2}$ values of $\operatorname{Tg} 2576$ mice were significantly lower than wild-type mice. In addition, the decline in $T_{2}$ in SCN of $\mathrm{Tg} 2576$ mice was clearly influenced by gender. In female Tg2576 mice, the decline in $T_{2}$ was considerably higher $(\mathrm{P}<0.001)$ than in male Tg2576 mice $(\mathrm{P}<$ 0.05). A $\beta$ plaques were not detected in SCN of Tg2576 mice (Supplementary Fig. 1).

Fig. 3 shows results of longitudinal monitoring of $T_{2}$ changes in the SCN of male and female $\operatorname{Tg} 2576$ and wild-type mice. In both wild-type male and wild-type female mice, there was no significant change in $T_{2}$ relaxation time between 10 and 18 months of age. At 10 months of age, $T_{2}$ values in the $\mathrm{SCN}$ of the wild-type and $\mathrm{Tg} 2576$ mice were not significantly different in both male and female mice. In male $\operatorname{Tg} 2576$ mice, the $T_{2}$ relaxation times show a trend to decrease with age. However, the difference between wild-type and Tg2576 mice was evident only at the age of 18 months. In contrast, female $\mathrm{Tg} 2576$ mice show a significant $T_{2}$ decrease in SCN as compared to wild-type mice at and above 12 month of age. The extent of decrease in $T_{2}$ in the $\mathrm{SCN}$ region of female $\mathrm{Tg} 2576$ mice relative to wild-type mice was $5.3 \%, 8.5 \%$ and $12.3 \%$ for 12,15 , and 18 months of age, respectively.

Results for NNLS analysis of the experimental decay curves are presented in Fig. 4. In SCN from wild-type mice two distinct components were consistently detected with NNLS. These were: short $T_{2}$ component $(7.04 \pm 0.1 \mathrm{~ms}$ in male and $5.47 \pm 2.3 \mathrm{~ms}$ in female) constituting < 
$0.1 \%$ of the total fraction, and an intermediate $T_{2}$ component $(27.6 \pm 0.94 \mathrm{~ms}$ in male and $27.19 \pm 1.02$ in female) constituting $>99 \%$ of the fraction.

Interestingly, in SCN of Tg2576 mice, NNLS analysis of the experimental decay curves shows three well-distinguished components, These are: short $T_{2}$ components $(17.43 \pm 3.04 \mathrm{~ms}$ in male and $10.46 \pm 2.38 \mathrm{~ms}$ in female) constituting $<3.5 \%$ of the fraction; intermediate $T_{2}$ components $(25.49 \pm 0.88 \mathrm{~ms}$ in male and $23.06 \pm 1.67$ in female) constituting $\sim 80 \%$ of the fraction and a long $T_{2}$ component $(67.59 \pm 6.26 \mathrm{~ms}$ in male and $81.66 \pm 8.52$ in female) constituting $>10-19 \%$ of the fraction. The intermediate component was reduced in $\operatorname{Tg} 2576$ mice while long $T_{2}$ component which is absent in SCN of wild-type mice is present in the SCN of $\mathrm{Tg} 2576$ mice.

Immunohistological analyses of number of neurons and astrocytes in SCN of male and female Tg2576 and wild-type mice are quantified in Figure 5. GFAP staining demonstrated significantly high numbers of activated astrocytes and NeuN staining rvealed a significantly decreased number of neurons in SCN of 18 month old Tg2576 mice as compared to agematched wild-type mice (Fig. 5). Thus an increase in the astrocytes-to-neurons ratio in SCN was clearly observed in Tg2576 as compared to wild-type mice (Fig. 5). The increase in astrocyte to neuron ratio was more pronounced in female than male mice (Fig. 5B).

The analysis of the expression level of glutamic acid decarboxylase (GAD), an enzyme involved in synthesizing GABA from glutamate in neurons is shown in Fig. 6. The level of GAD was significantly lower in the SCN of 18 month old Tg2576 mice as compared to agedmatched wild-type mice (Fig. 6). The decline in GAD was more prominent in female as compared to male $\operatorname{Tg} 2576$ mice, and it was inversely allied with high number of astrocytes in SCN of female as compared to male mice (Fig. 5B). 


\section{DISCUSSION}

Several studies have suggested that loss of integrity and dysfunction of the SCN lies at the root of the circadian dysfunction which may be the cause or the consequence of $\mathrm{AD}$ pathogenesis. Knowledge on how and when these SCN integrity changes occur would help to find out whether the circadian dysfunction is the cause or the consequence of $\mathrm{AD}$ pathogenesis. Therefore, in this study, longitudinal magnetic resonance $T_{2}$ relaxation measurements were used to probe subtle microstructural changes in the SCN in vivo.

The $T_{2}$ values of various grey matter regions such as the hippocampus, cingulate and piriform cortex of the 18 month old $\mathrm{Tg} 2576$ mice showed a significant decrease as compared to the age-matched wild-type mice. These results are in agreement with previous studies, which showed a prominent decrease in $T_{2}$ for $\mathrm{A} \beta$ plaque-rich regions such as the hippocampus and cortex of Tg2576 mice as compared to age-matched wild-type mice. Although the source of the $T_{2}$ reduction in various grey matter regions remains unclear, previous studies have suggested that iron-associated $\mathrm{A} \beta$ plaques may be involved in reducing the $T_{2}$ in $\mathrm{AD}$ brain [23]. Interestingly, the decline in hippocampal $T_{2}$ relaxation time was more significant in female as compared to male $\operatorname{Tg} 2576$ mice. These results are in line with higher $A \beta$ plaque load in hippocampus of female mice as compared to male mice seen in our previous studies [37]. On the other hand, in the thalamus region, which was associated with very low $A \beta$ deposition [30], no significant change in $T_{2}$ values between wild-type and $\operatorname{Tg} 2576$ mice was observed. Interestingly, $T_{2}$ values in the $\mathrm{SCN}$ region of $\mathrm{Tg} 2576$ mice were significantly lower than wild-type mice. In addition, the decline in $T_{2}$ in $\mathrm{SCN}$ of $\operatorname{Tg} 2576$ mice was clearly influenced by gender. Unlike in hippocampus and cortex regions, A $\beta$ plaques were not detected in SCN of Tg2576 mice (Supplementary Fig. 1). 
To get further inside into the gender-specific effects on $T_{2}$ in $\mathrm{SCN}$, we performed a longitudinal in vivo study to follow the change in $T_{2}$ time with age in wild-type and Tg2576 mice. While there was no significant change in $T_{2}$ relaxation time between 10 and 18 months of age in wild type mice, the $T_{2}$ relaxation times show a trend to decrease with age in Tg2576 mice. In males, the significant difference between wild-type and Tg2576 mice was evident only at the age of 18 months, however, in females, the difference between wild-type and Tg2576 mice was evident at 12 month of age already.

$T_{2}$ is influenced by the mobility of water molecules associated with various effective tissue compartments, defined by intracellular and extracellular macromolecular components, which would be expected to exhibit a distinct $T_{2}$ [38]. In previous studies, multiexponential $T_{2}$ analysis has been successfully applied to detect and characterize multiple water compartments associated with tissue microstructures in normal and pathologic tissue [19, 27, 38-41]. Consistent with previous work in other tissues, we expect this approach to lead to an insight into water compartmentation in SCN and how they are influenced during disease progression. We further assume that the ability of multiexponential analysis to detect AD related changes in water compartmentation in SCN would support its use as a potential diagnostic approach in early sleep disturbance in $\mathrm{AD}$. Therefore, in this work, we investigated water compartmentation in SCN under normal and $\mathrm{AD}$-like conditions using multiexponential analysis of $T_{2}$ relaxation data. This analysis was performed using nonnegative least squares (NNLS) algorithm. In SCN from wild-type mice two distinct components were detected which include a short $T_{2}$ component constituting $<0.1 \%$ of the total fraction, and an intermediate $T_{2}$ component constituting $>99 \%$ of the fraction. On the other hand, in SCN of Tg2576 mice, we observed three well-distinguished components namely: a short $T_{2}$ components constituting $<3.5 \%$ of the fraction; an intermediate $T_{2}$ component constituting $80 \%$ of the fraction and a long $T_{2}$ component constituting $>10-19 \%$ of the fraction. 
The short $T_{2}$ component with smallest contribution in the signal with $T_{2}=6 \mathrm{~ms}$ in wild-type and $T_{2}=13 \mathrm{~ms}$ in $\mathrm{Tg} 2576$ could be attributed to the protein bound water as has been proposed previously [42]. The amplitude of this fast relaxing component, calculated with NNLS algorithm, is higher in SCN of $\mathrm{Tg} 2576$ mice, suggesting an increase in the protein bound water. Intermediate $T_{2}$ component with largest contribution in the signal can be assigned to the intra or extracellular water in the SCN region of the brain. Signal intensity, calculated with NNLS algorithm, of this component is largely reduced in Tg2576 mice and this reduction was more severe in female than male $\operatorname{Tg} 2576$ mice. The $T_{2}$ value of the intermediate components was also decreased in Tg2576 mice as compared to wild-type mice, and this decrease was also more severe in female mice than male mice.

Long $T_{2}$ component was present only in Tg2576 but not in wild-type mice. The existence of this slow relaxing component in Tg2576 may either hints towards inflammation, axonal loss, and/or demyelination. However, since SCN contains small, densely packed neurons, and is largely devoid of myelinated fibers [43], the appearance of long $T_{2}$ component in SCN of $\operatorname{Tg} 2576$ mice may not be a manifestation of demyelination. On the other hand, inflammation commonly occurs in a wide spectrum of nervous system diseases including AD. Inflammation has been known to results in an increase in extracellular water volume fraction. This in turn leads to an increase in long $T_{2}$ component as described by Stanisz et al. [27]. In mice, it has been demonstrated that neuroinflammation plays a role in the functional and molecular changes that the SCN undergoes during senescence [44]. However, little evidence for neuroinflammatory processes in the SCN of AD patients is currently available [45].

In our study, histological analyses show significantly elevated numbers of activated astrocytes in SCN of Tg2576 mice. In contrast, the neuronal numbers were significantly decreased in SCN of 18 month old Tg2576 mice. Thus an increase in the ratio of astrocytes to neurons in 
SCN was clearly observed in Tg2576 as compared to wild-type mice (Fig. 5). These results are in line with human brain studies where an increase in astrocyte to neuron ratio has been observed in SCN of AD subjects [46]. Interestingly, in our study, the increase in astrocyte to neuron ratio was more substantial in female than male mice (Fig. 5B).

When glial cells are activated, common inflammatory agents such as pro-inflammatory cytokines and reactive oxygen species, secreted from these cells, can lead to inflammatory response and contribute to the disease progression [47]. Astrocytes play an important role in water homeostasis. When they are activated, the expression of astrocytic proteins such as aquaporin-4 (AQP4) is reduced which leads to abnormalities in membrane water transport $[48,49]$. Subsequently, these abnormalities might lead to an increase in extracellular fluid leading to an increase in the long $T_{2}$ component in $\mathrm{SCN}$ of $\mathrm{Tg} 2576$ mice, as observed in our study.

The neurotransmitter $\gamma$-aminobutyric acid (GABA) and its receptors are widely expressed in the SCN where they mediate cell- cell communication $[3,6]$. The role of GABA in inhibiting pro-inflammatory responses has been well recognized $[50,51]$. To explore whether an increase in inflammatory response in SCN of transgenic mice may be associated with low levels of GABA production in neurons, we have analysed the expression level of glutamic acid decarboxylase (GAD), an enzyme involved in synthesizing GABA from glutamate in neurons $[52,53]$. A significantly lower level of GAD was observed in SCN regions of 18 month old Tg2576 mice as compared to aged-matched wild-type mice (Fig. 6). The decline in GAD was more significant in female as compared to male Tg2576 mice. which is inversely correlated with high number of astrocytes in SCN of female as compared to male mice (Fig. 5B). These results indicate that low GABA production in $\mathrm{SCN}$ and consequently an inefficient GABA signaling may lead to proinflammatory cytokine production and 
inflammatory responses which are reflected with an increase in long $T_{2}$ components in SCN of $\operatorname{Tg} 2576$ mice. In other brain regions such as hippocampus and cortex, astrocytes and microglia become activated close to senile plaques (Supplementary Fig. 1) in order to internalize and degrade $A \beta$ [54]. Since in SCN senile plaques were not present, the activated astrocytes were independent/unrelated with senile plaques. Future studies, on exploring GABA production specifically in activated astrocytes of $\mathrm{SCN}$ and its relation with $\mathrm{AD}$ progression would shed more light on the mechanism of SCN dysfunction in AD.

In conclusion, our results suggest that gender-specific differences in inflammatory response, most likely manifested by low GABA production, occur in SCN of Tg2576 mice. This may be an underlying perpetrator for the changes in circadian rhythmicity and sleep disturbance in $\mathrm{AD}$ and could also be at the root of marked sex disparities observed in $\mathrm{AD}$ subjects. Our results also demonstrate the ability of in vivo multiexponential $T_{2}$ analysis to detect ADrelated subtle microstructural changes in SCN and support its use as a potential non-invasive diagnostic approach in early sleep disturbance in $\mathrm{AD}$.

\section{ACKNOWLEDGEMENTS}

This work was partly supported by grants from Alzheimer Forschung Initiative e.V. (AFI, Grant Nr 13810). We are grateful to Dr. Karen Hsiao Ashe (University of Minnesota, USA) for providing the initial Tg2576 mice for further breeding. We thank Dr. Firat Kara and Dr. Niels Braakman for providing expert help for $T_{2}$ experiments.

\section{CONFLICT OF INTEREST/DISCLOSURE STATEMENT}

The authors have no conflict of interest to report. 


\section{REFERENCES}

[1] Welsh DK, Takahashi JS, Kay SA (2010) Suprachiasmatic nucleus: cell autonomy and network properties. Annu Rev Physiol 72, 551-577.

[2] van Oosterhout F, Lucassen EA, Houben T, vanderLeest HT, Antle MC, Meijer JH (2012) Amplitude of the SCN clock enhanced by the behavioral activity rhythm. PloS one 7, e39693e39693.

[3] DeWoskin D, Myung J, Belle MDC, Piggins HD, Takumi T, Forger DB (2015) Distinct roles for GABA across multiple timescales in mammalian circadian timekeeping. Proc Natl Acad Sci U S A 112, E3911-E3919.

[4] Farajnia S, van Westering TLE, Meijer JH, Michel S (2014) Seasonal induction of GABAergic excitation in the central mammalian clock. Proc Natl Acad Sci USA 111, $9627-9632$.

[5] Albus H, Vansteensel MJ, Michel S, Block GD, Meijer JH (2005) A GABAergic Mechanism Is Necessary for Coupling Dissociable Ventral and Dorsal Regional Oscillators within the Circadian Clock. Curr Biol 15, 886-893.

[6] Liu C, Reppert SM (2000) GABA Synchronizes Clock Cells within the Suprachiasmatic Circadian Clock. Neuron 25, 123-128.

[7] Moore RY, Speh JC (1993) GABA is the principal neurotransmitter of the circadian system. Neurosci Lett 150, 112-116.

[8] Wagner S, Castel M, Gainer H, Yarom Y (1997) GABA in the mammalian suprachiasmatic nucleus and its role in diurnal rhythmicity. Nature 387, 598.

[9] Musiek ES, Xiong DD, Holtzman DM (2015) Sleep, circadian rhythms, and the pathogenesis of Alzheimer disease. Exp Mol Med 47, e148-e148. 
[10] Roozendaal B, van Gool WA, Swaab DF, Hoogendijk JE, Mirmiran M (1987) Changes in vasopressin cells of the rat suprachiasmatic nucleus with aging. Brain Res 409, 259-264.

[11] Swaab DF, Fliers E, Partiman TS (1985) The suprachiasmatic nucleus of the human brain in relation to sex, age and senile dementia. Brain Research 342, 37-44.

[12] Zhou J-N, Hofman MA, Swaab DF (1995) VIP neurons in the human SCN in relation to sex, age, and Alzheimer's disease. Neurobiol of Aging 16, 571-576.

[13] Okawa M, Mishima K, Hishikawa Y, Hozumi S, Hori H, Takahashi K (1991) Circadian Rhythm Disorders in Sleep-Waking and Body Temperature in Elderly Patients with Dementia and Their Treatment. Sleep 14, 478-485.

[14] Sterniczuk R, Dyck RH, LaFerla FM, Antle MC (2010) Characterization of the 3xTg-AD mouse model of Alzheimer's disease: Part 1. Circadian changes. Brain Res 1348, 139-148.

[15] Wisor JP, Edgar DM, Yesavage J, Ryan HS, McCormick CM, Lapustea N, Murphy GM (2005) Sleep and circadian abnormalities in a transgenic mouse model of Alzheimer's disease: A role for cholinergic transmission. Neuroscience 131, 375-385.

[16] Lloret A, Badía M-C, Mora NJ, Ortega A, Pallardó FV, Alonso M-D, Atamna H, Viña J (2008) Gender and age-dependent differences in the mitochondrial apoptogenic pathway in Alzheimer's disease. Free Radical Biol Med 44, 2019-2025.

[17] van Duijn S, Nabuurs RJA, van Duinen SG, Natté R, van Buchem MA, Alia A (2013) Longitudinal monitoring of sex-related in vivo metabolic changes in the brain of Alzheimer's disease transgenic mouse using magnetic resonance spectroscopy. J Alzheimer's Dis 34, 10511059.

[18] Carter CL, Resnick EM, Mallampalli M, Kalbarczyk A (2012) Sex and Gender Differences in Alzheimer's Disease: Recommendations for Future Research. J Womens Health 21, 10181023. 
[19] MacKay A, Laule C, Vavasour I, Bjarnason T, Kolind S, Mädler B (2006) Insights into brain microstructure from the T2 distribution. Magn Reson Imaging 24, 515-525.

[20] Miot-Noirault E, Barantin L, Akoka S, Le Pape A (1997) T2 relaxation time as a marker of brain myelination: experimental MR study in two neonatal animal models. J Neurosci Methods 72, 5-14.

[21] Helpern JA, Lee S-P, Falangola MF, Dyakin VV, Bogart A, Ardekani B, Duff K, Branch C, Wisniewski T, de Leon MJ, Wolf O, O'Shea J, Nixon RA (2004) MRI assessment of neuropathology in a transgenic mouse model of Alzheimer's disease. Magn Reson Med 51, 794-798.

[22] Kara F, Höfling C, Roßner S, Schliebs R, Linden AVd, Groot HJM, Alia A (2015) In Vivo Longitudinal Monitoring of Changes in the Corpus Callosum Integrity During Disease Progression in a Mouse Model of Alzheimer's Disease. Curr Alzheimer Res 12, 941-950.

[23] Falangola MF, Dyakin VV, Lee SP, Bogart A, Babb JS, Duff K, Nixon R, Helpern JA (2007) Quantitative MRI reveals aging-associated T2 changes in mouse models of Alzheimer's disease. NMR Biomed 20, 343-351.

[24] Kumar R, Delshad S, Woo MA, Macey PM, Harper RM (2012) Age-related regional brain T2-relaxation changes in healthy adults. J Magn Reson Imaging 35, 300-308.

[25] Kumar R, Chavez AS, Macey PM, Woo MA, Harper RM (2013) Brain axial and radial diffusivity changes with age and gender in healthy adults. Brain res 1512, 22-36.

[26] Robert W. Thatcher, Duane M. North, Richard T. Curtin, Rebecca A. Walker, Carl J. Biver, Juan F. Gomez, Andres M. Salazar (2001) An EEG Severity Index of Traumatic Brain Injury. J Neuropsychiatry Clin Neurosci 13, 77-87.

[27] Stanisz GJ, Webb S, Munro CA, Pun T, Midha R (2004) MR properties of excised neural tissue following experimentally induced inflammation. Magn Reson Med 51, 473-479. 
[28] Hsiao K, Chapman P, Nilsen S, Eckman C, Harigaya Y, Younkin S, Yang F, Cole G (1996) Correlative Memory Deficits, A $\beta$ Elevation, and Amyloid Plaques in Transgenic Mice. Science 274, 99-103.

[29] Kent BA, Strittmatter SM, Nygaard HB (2018) Sleep and EEG Power Spectral Analysis in Three Transgenic Mouse Models of Alzheimer's Disease: APP/PS1, 3xTgAD, and Tg2576. $J$ Alzheimer's Dis 64, 1325-1336.

[30] Braakman N, Matysik J, van Duinen SG, Verbeek F, Schliebs R, de Groot HJM, Alia A (2006) Longitudinal assessment of Alzheimer's $\beta$-amyloid plaque development in transgenic mice monitored by in vivo magnetic resonance microimaging. J Magn Reson Imaging 24, $530-536$

[31] Meiboom S, Gill D (1958) Modified Spin-Echo Method for Measuring Nuclear Relaxation Times. Rev Sci Instrum 29, 688-691.

[32] Carr HY, Purcell EM (1954) Effects of Diffusion on Free Precession in Nuclear Magnetic Resonance Experiments. Physl Rev 94, 630-638.

[33] Hennig J, Nauerth A, Friedburg H (1986) RARE imaging: A fast imaging method for clinical MR. Magn Reson Med 3, 823-833.

[34] Thiessen JD, Zhang Y, Zhang H, Wang L, Buist R, Del Bigio MR, Kong J, Li X-M, Martin M (2013) Quantitative MRI and ultrastructural examination of the cuprizone mouse model of demyelination. NMR Biomed 26, 1562-1581.

[35] Müller R, Büttner P (1994) A critical discussion of intraclass correlation coefficients. Stat Med 13, 2465-2476.

[36] Bartzokis G, Mintz J, Marx P, Osborn D, Gutkind D, Chiang F, Phelan CK, Marder SR (1993) Reliability of in vivo volume measures of hippocampus and other brain structures using MRI. Magn Reson Imaging 11, 993-1006. 
[37] Roy U, Stute L, Höfling C, Hartlage-Rübsamen M, Matysik J, Roßner S, Alia A (2018) Sexand age-specific modulation of brain GABA levels in a mouse model of Alzheimer's disease. Neurobiol Aging 62, 168-179.

[38] Reiter DA, Lin P-C, Fishbein KW, Spencer RG (2009) Multicomponent T2 relaxation analysis in cartilage. Magn Reson Med 61, 803-809.

[39] Ababneh Z, Beloeil H, Berde CB, Gambarota G, Maier SE, Mulkern RV (2005) Biexponential parameterization of diffusion and T2 relaxation decay curves in a rat muscle edema model: Decay curve components and water compartments. Magn Reson Med 54, 524-531.

[40] Kolind SH, Laule C, Vavasour IM, Li DKB, Traboulsee AL, Mädler B, Moore GRW, MacKay AL (2008) Complementary information from multi-exponential T2 relaxation and diffusion tensor imaging reveals differences between multiple sclerosis lesions. NeuroImage 40, 77-85.

[41] Peled S, Cory DG, Raymond SA, Kirschner DA, Jolesz FA (1999) Water diffusion, T(2), and compartmentation in frog sciatic nerve. Magn Reson Med 42, 911-918.

[42] Stankeiwicz PJ, Metz KR, Sassani JW, Briggs RW (1989) Nuclear magnetic resonance study of free and bound water fractions in normal lenses. Invest Ophthalmol Vis Sci 30, 2361-2369.

[43] Tessonneaud A, Cooper HM, Caldani M, Locatelli A, Viguier-Martinez M-C (1994) The suprachiasmatic nucleus in the sheep: retinal projections and cytoarchitectural organization. Cell Tissue Res 278, 65-84.

[44] Bentivoglio M, Deng XH, Nygård M, Sadki A, Kristensson K (2006) The aging suprachiasmatic nucleus and cytokines: functional, molecular, and cellular changes in rodents. Chronobiol Int 23, 437-449.

[45] Van Erum J, Van Dam D, De Deyn PP (2018) Sleep and Alzheimer's disease: A pivotal role for the suprachiasmatic nucleus. Sleep Med Rev 40, 17-27. 
[46] Stopa EG, Volicer L, Kuo-Leblanc V, Harper D, Lathi D, Tate B, Satlin A (1999) Pathologic Evaluation of the Human Suprachiasmatic Nucleus in Severe Dementia. J Neuropathol Exp Neurol 58, 29-39.

[47] Rubio-Perez JM, Morillas-Ruiz JM (2012) A review: inflammatory process in Alzheimer's disease, role of cytokines. TheScientificWorldJournal 2012, 756357-756357.

[48] Gunnarson E, Axehult G, Baturina G, Zelenin S, Zelenina M, Aperia A (2005) Lead induces increased water permeability in astrocytes expressing aquaporin 4. Neuroscience 136, 105114.

[49] Yang W, Wu Q, Yuan C, Gao J, Xiao M, Gu M, Ding J, Hu G (2012) Aquaporin-4 mediates astrocyte response to $\beta$-amyloid. Mol Cell Neurosci 49, 406-414.

[50] Lee M, Schwab C, Mcgeer PL (2011) Astrocytes are GABAergic cells that modulate microglial activity. Glia 59, 152-165.

[51] Prud'homme GJ, Glinka Y, Hasilo C, Paraskevas S, Li X, Wang Q (2013) GABA Protects Human Islet Cells Against the Deleterious Effects of Immunosuppressive Drugs and Exerts Immunoinhibitory Effects Alone. Transplantation 96, 616-623.

[52] McQuail JA, Frazier CJ, Bizon JL (2015) Molecular aspects of age-related cognitive decline: the role of GABA signaling. Trends Mol Med 21, 450-460.

[53] Stanley DP, Shetty AK (2004) Aging in the rat hippocampus is associated with widespread reductions in the number of glutamate decarboxylase-67 positive interneurons but not interneuron degeneration. J Neurochem 89, 204-216.

[54] Mohamed A, Posse de Chaves E (2011) A $\beta$ Internalization by Neurons and Glia. Int J Alzheimers Dis 2011, 17. 


\section{FIGURE LEGENDS}

Fig 1. (A) Anatomical $T_{2}$-weighted MR coronal slices of a mouse brain, showing various brain regions for $T_{2}$ measurements acquired with the RARE sequence at $17.6 \mathrm{~T}$. Scale bar, $750 \mu \mathrm{m}$ (B) Test-retest reliability of in vivo $T_{2}$ measurements in a variety of brain regions performed by intra-class correlation coefficient $\left(\mathrm{ICC}_{2,1}\right)$ using a 2-way random effects ANOVA (subject by session) and the absolute agreement definition. There is a one-day interval between session 1 and session 2 measurements performed on the same mice. $T_{2}$ values are presented as means from five subjects $\pm \mathrm{SD}$; Paired t-test results collected from the same mice in two sessions show no significant (NS) difference in all cases $(\mathrm{P}>0.05, \mathrm{n}=5)$. (C) Effect of refocusing interpulse interval $(\tau)$ on T2 in the suprachiasmatic nucleus (SCN) and hippocampus $(\mathrm{HC})$ regions. $T_{2}$ values were measured using the multi-slice multi-echo sequence with 4 different $\tau$ values namely $6.1,12.2,18.3,24.4,30.5$ and 36.6 ms. Values are expressed as mean $T_{2}$ in $\mathrm{ms} \pm$ standard deviation (error bars); $\mathrm{n}=3$. Abbreviations: cingulate cortex (CG), piriform cortex (PIR), hippocampus (HC) and suprachiasmatic nucleus (SCN).

Fig. 2. In vivo $T_{2}$ relaxation time changes in the cingulate cortex (CG), piriform cortex (PIR), hippocampus $(\mathrm{HC})$ and suprachiasmatic nucleus $(\mathrm{SCN})$ regions of a wild-type female (WT

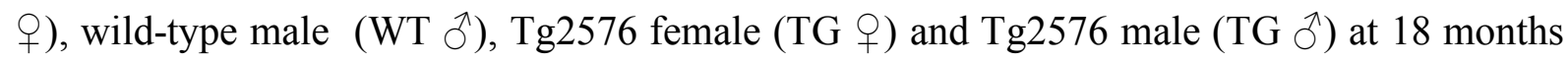
of age. Data represent the mean $T_{2}$ in $\mathrm{ms} \pm \mathrm{SD}$ (error bars) $(95 \%$ C.I.) of $\mathrm{n}=6$, except for $\mathrm{TG}$ §: $\mathrm{n}=4$. Student $\mathrm{t}-$ test: ${ }^{*} \mathrm{p}<0.05, * * \mathrm{p}<0.01, \# \mathrm{p}<0.001$, statistically significant.

Fig. 3. Gender-specific, age-dependent in vivo $T_{2}$ changes of the suprachiasmatic nucleus (SCN) region of the wild-type (WT) and $\mathrm{Tg} 2576(\mathrm{TG})$ mice. Values are expressed as mean $T_{2}$ in $\mathrm{ms} \pm \mathrm{SD}$ (error bars) $(95 \%$ C.I.). Two tailed student t- test show no significant difference observed between WT and TG mice at the age of 10 months $(\mathrm{P}>0.05)$ in individual gender groups. Significant difference between WT and TG mice at the age of 12 months, 15 months 
and 18 months were observed in female mice but in male mice decline in $T_{2}$ relaxation is observed only at 18 month of age. However, female mice has steeper decline in $T_{2}$ relaxation compared to TG male mice. Student t- test: ${ }^{*} \mathrm{p}<0.05, * * \mathrm{p}<0.01$

Fig. 4. Multicomponent analysis of the experimental $T_{2}$ relaxation decay curves using a nonnegative least square based algorithm (NNLS). (A) NNLS least-squares spectra of SCN regions of a wild-type male (WT §), wild-type female (WT $ᄋ$ ), $\operatorname{Tg} 2576$ male (TG $\precsim$ ) and Tg2576 female (TG ) ) at 18 months of age. Spectra show difference in the amplitude and time of various relaxation components between WT and TG which were also influenced by gender. (B) Time and amplitude of various $T_{2}$ components are summarized in the table. Intermediate $T_{2}$ components show largest contribution while short $T_{2}$ components with smallest contribution in the signal. Long $T_{2}$ component was only present in the SCN region of TG mice and was absent in the WT mice.

Fig. 5. Immunohistochemical analyses of numbers of neurons and astrocytes in SCN of male and female Tg2576 and WT mice. (A) Representative confocal images of GFAP (red) and NeuN (cyan) stained sections through SCN of TG mice. Scale bar, $250 \mu \mathrm{m}$ (and $60 \mu \mathrm{m}$ in magnifications). (B) Quantitative analysis of neuronal cells (left), astrocytes (middle) and ratio of astrocytes to neuronal cells in SCN of 18 months old male (ð) and female () WT and Tg2576 (TG) mice. ${ }^{*} \mathrm{p}<0.05,{ }^{*} \mathrm{p}<0.05$. Abbreviation: NeuN, Neuronal Nuclei; GFAP, glial fibrillary acidic protein.

Fig. 6. Immunohistochemical analyses of GAD and GFAP staining in SCN of Tg2576 and WT mice. (A) Representative confocal images of GAD (blue) and GFAP (red) stained sections through SCN of 18 month old WT and TG mice. Scale bar, $250 \mu \mathrm{m}$ (and $60 \mu \mathrm{m}$ in magnifications). (B) Quantitative analysis of GAD staining in SCN of 6 and 18 months old 
male $\left({ }^{\Uparrow}\right)$ and female () WT and Tg2576 (TG) mice. ${ }^{* *} \mathrm{p}<0.05,{ }^{*} \mathrm{p}<0.05$. Abbreviation: GFAP, glial fibrillary acidic protein; GAD, glutamic acid decarboxylase. 
A
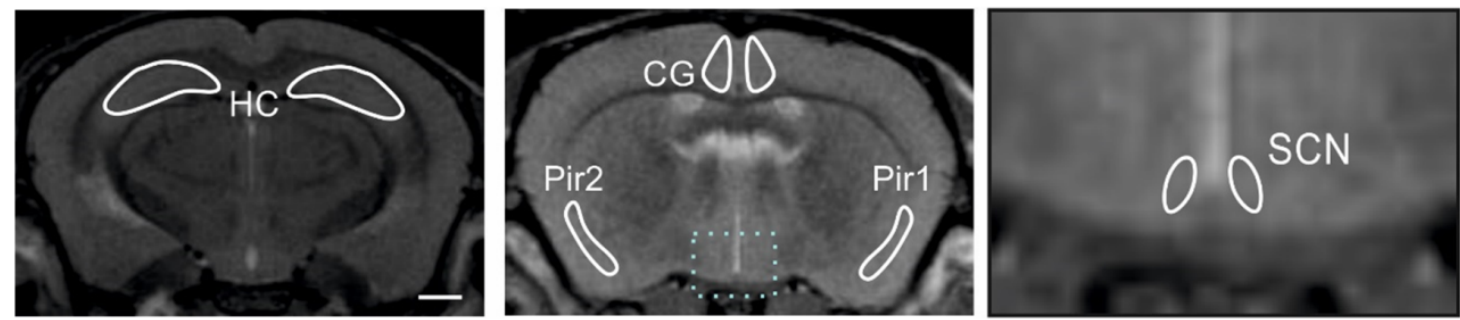

B

\begin{tabular}{ccccc}
\hline \multirow{2}{*}{ Structure } & \multicolumn{2}{c}{ Mean \pm SD } & \multirow{2}{*}{ ICC $_{2,1}$} & \multirow{2}{*}{ Paired t test } \\
\cline { 2 - 3 } & Session 1 & Session 2 & & \\
\hline CG & $28.98 \pm 0.38$ & $28.29 \pm 0.32$ & 0.75 & $\mathrm{~ns}$ \\
HC & $29.61 \pm 0.55$ & $30.01 \pm 0.78$ & 0.66 & $\mathrm{~ns}$ \\
PIR & $29.06 \pm 0.75$ & $28.85 \pm 0.81$ & 0.72 & $\mathrm{~ns}$ \\
SCN & $27.57 \pm 0.51$ & $27.58 \pm 0.61$ & 0.73 & $\mathrm{~ns}$ \\
\hline
\end{tabular}

C

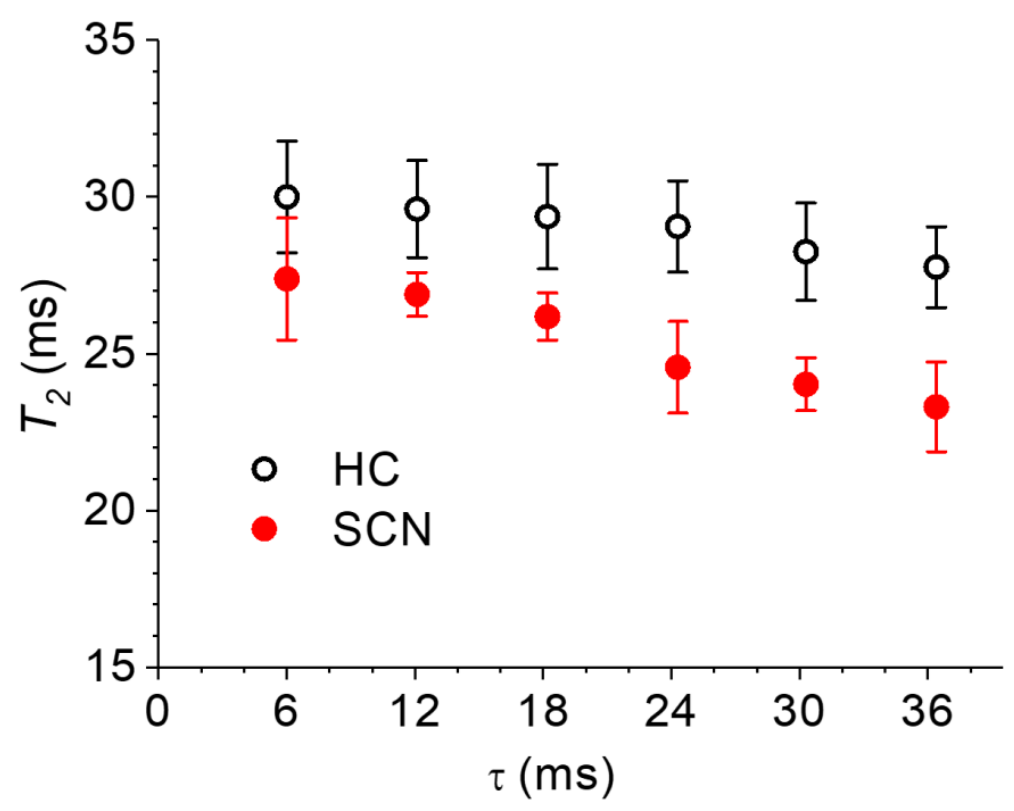

Fig. 1 


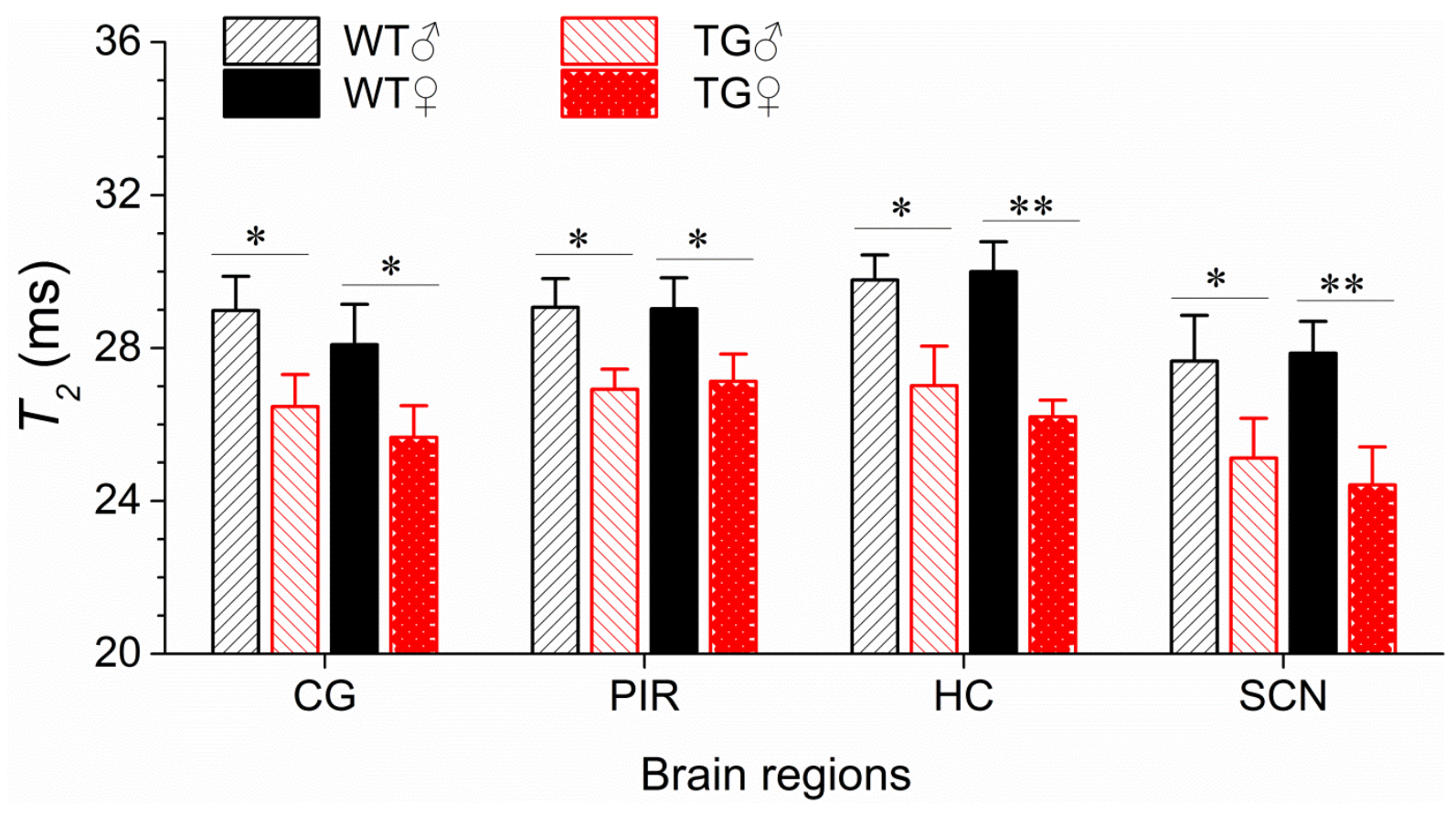

Fig. 2 
A

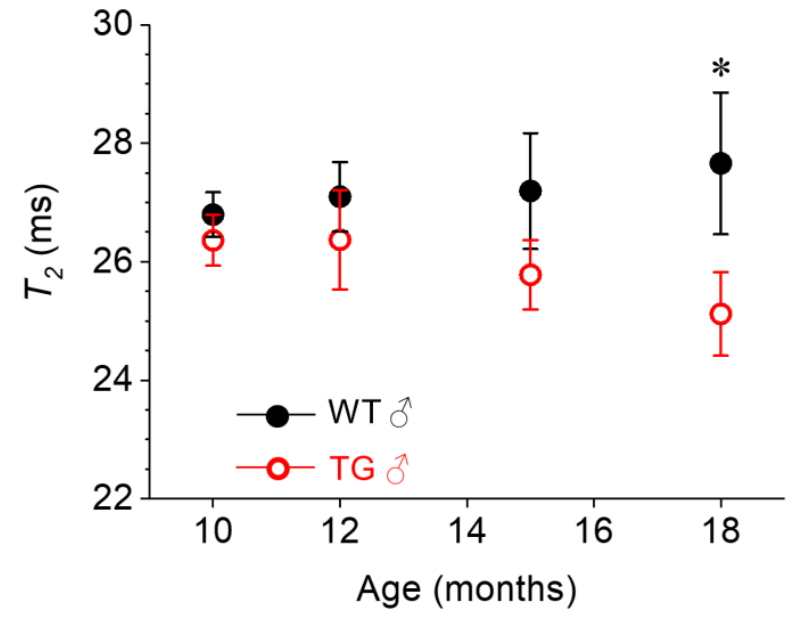

B

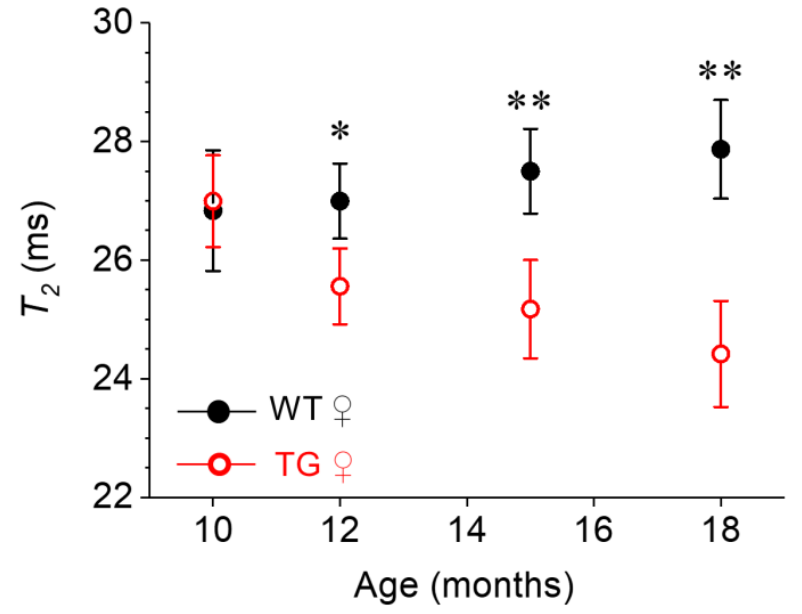

Fig. 3 

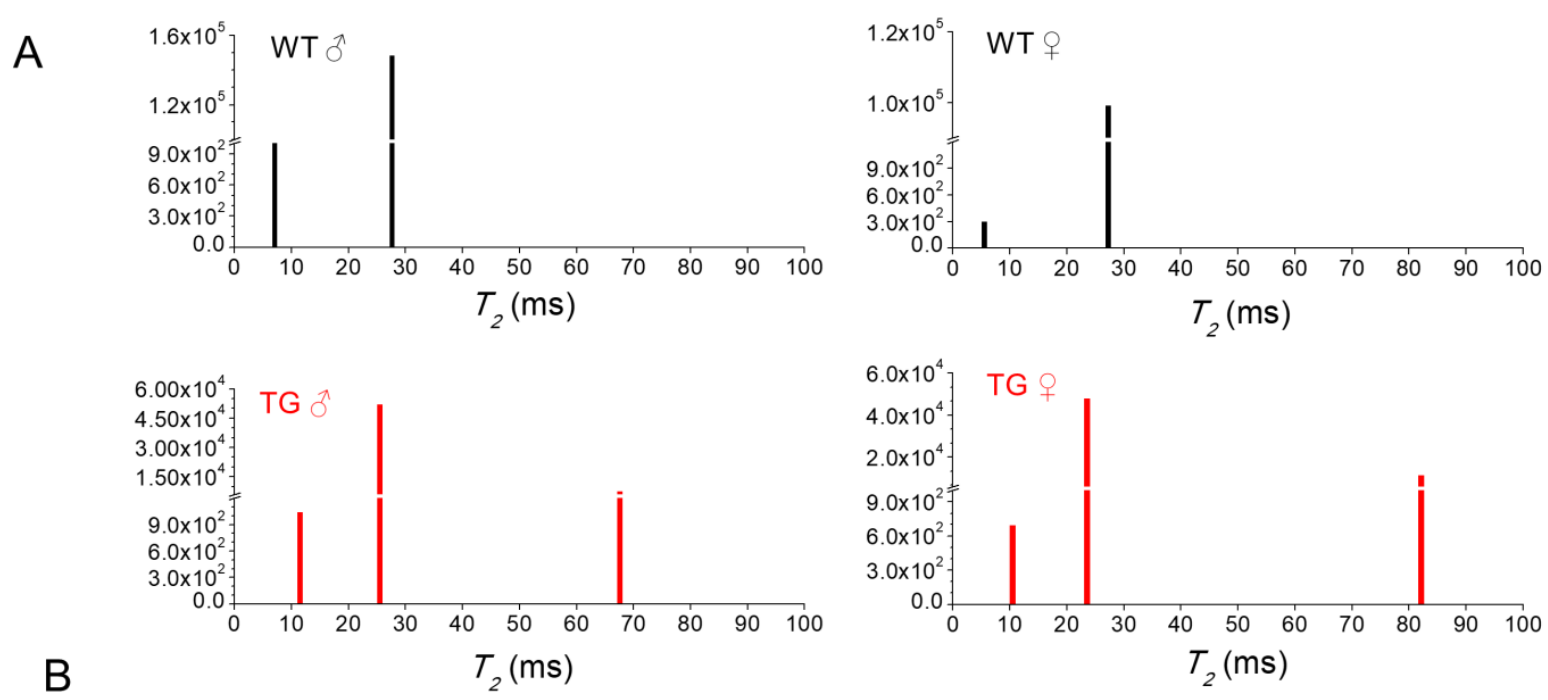

\begin{tabular}{|c|c|c|c|c|c|c|c|c|}
\hline & \multicolumn{2}{|c|}{ WT on } & \multicolumn{2}{|c|}{ TG ơ } & \multicolumn{2}{|c|}{ WT 9} & \multicolumn{2}{|c|}{ TG 9} \\
\hline & $\begin{array}{c}\mathrm{T}_{2} \\
(\mathrm{msec})\end{array}$ & $\begin{array}{c}\text { Amplitude } \\
(\%)\end{array}$ & $\begin{array}{c}\mathrm{T}_{2} \\
\text { (msec) }\end{array}$ & $\begin{array}{c}\text { Amplitude } \\
(\%)\end{array}$ & $\begin{array}{c}\mathrm{T}_{2} \\
\text { (msec) }\end{array}$ & $\begin{array}{c}\text { Amplitude } \\
(\%)\end{array}$ & $\begin{array}{c}\mathrm{T}_{2} \\
\text { (msec) }\end{array}$ & $\begin{array}{c}\text { Amplitude } \\
(\%)\end{array}$ \\
\hline Short component & $7.04 \pm 1.07$ & 0.1 & $17.43 \pm 3.04$ & 3.4 & $5.47 \pm 2.3$ & 0.9 & $10.46 \pm 2.38$ & 1.15 \\
\hline Intermediate component & $27.60 \pm 0.94$ & 99.9 & $25.49 \pm 0.88$ & 86.4 & $27.19 \pm 1.02$ & 99.1 & $23.06 \pm 1.67$ & 79.8 \\
\hline Long component & - & & $67.59 \pm 6.26$ & 10.2 & - & & $81.66 \pm 8.52$ & 19.0 \\
\hline
\end{tabular}

Fig. 4 

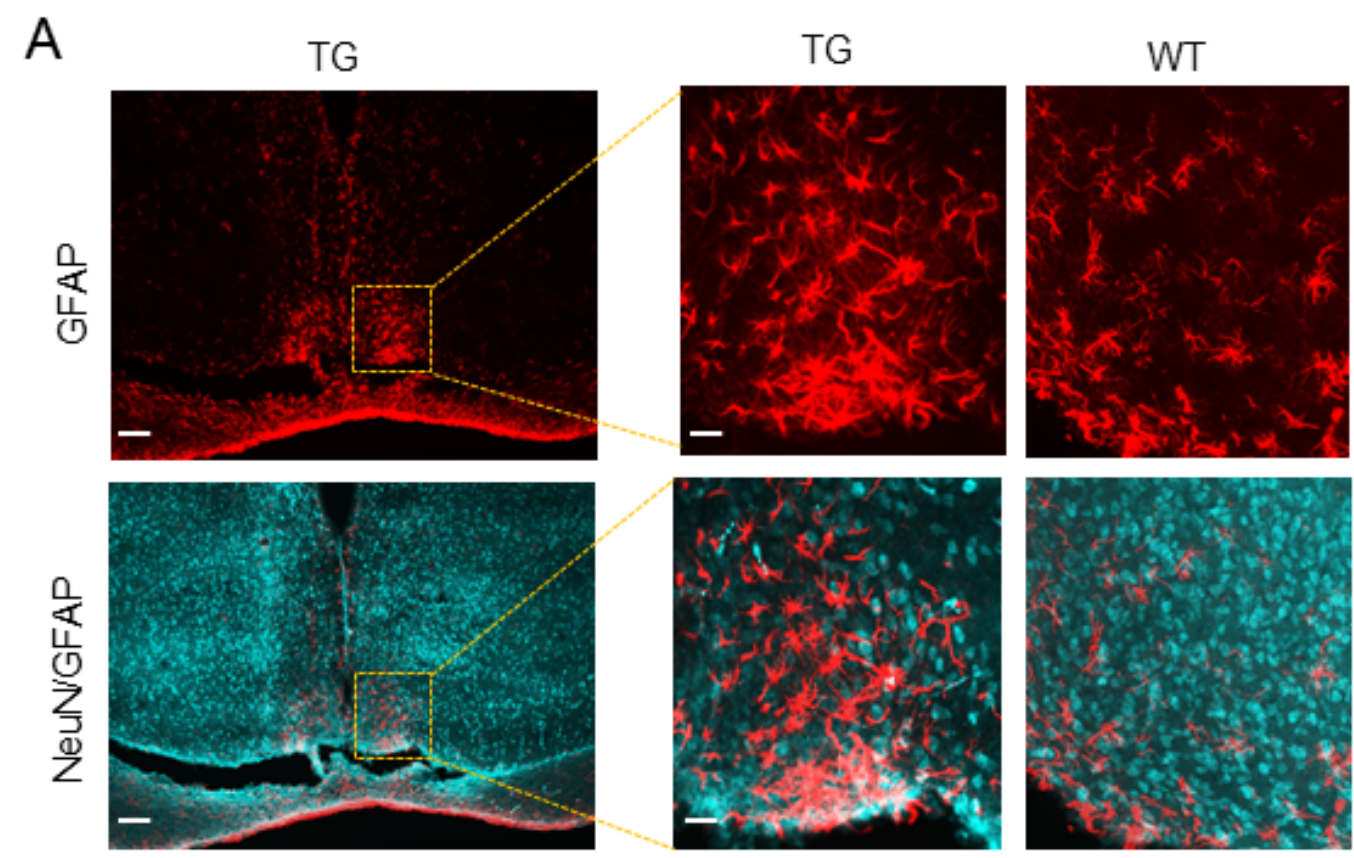

B
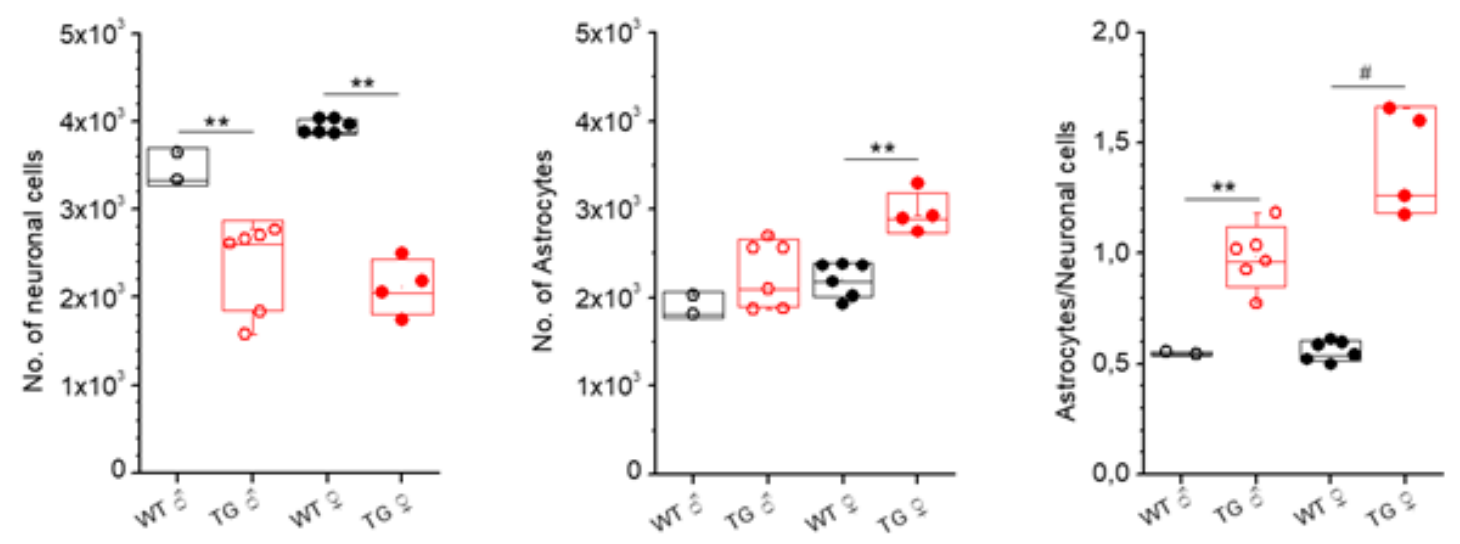

Fig. 5 


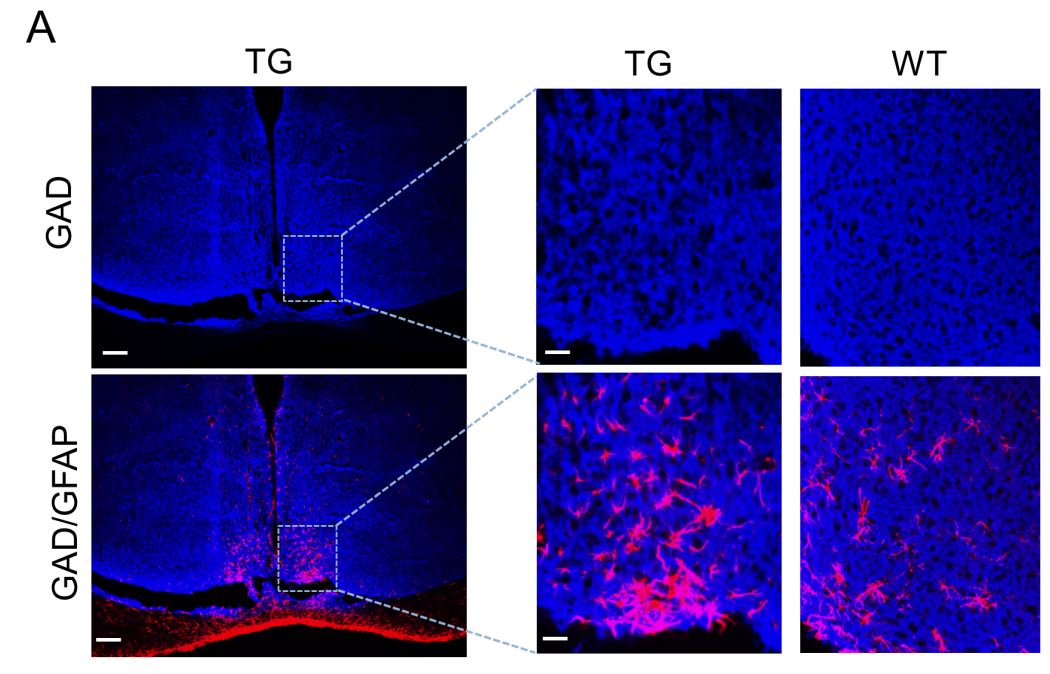

B

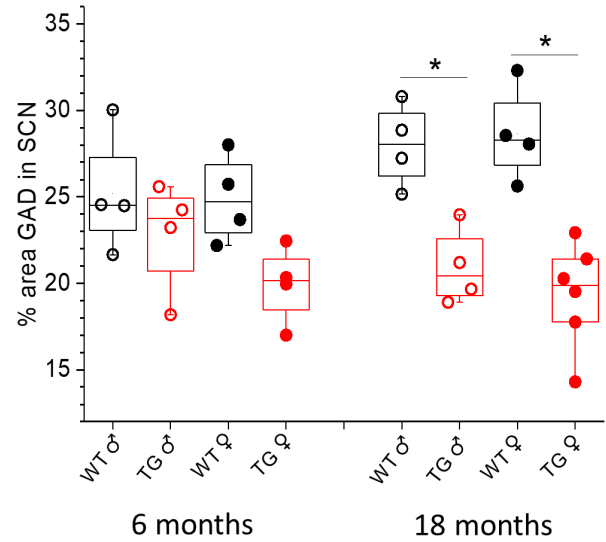

Fig. 6 


\section{Supplementary Information}
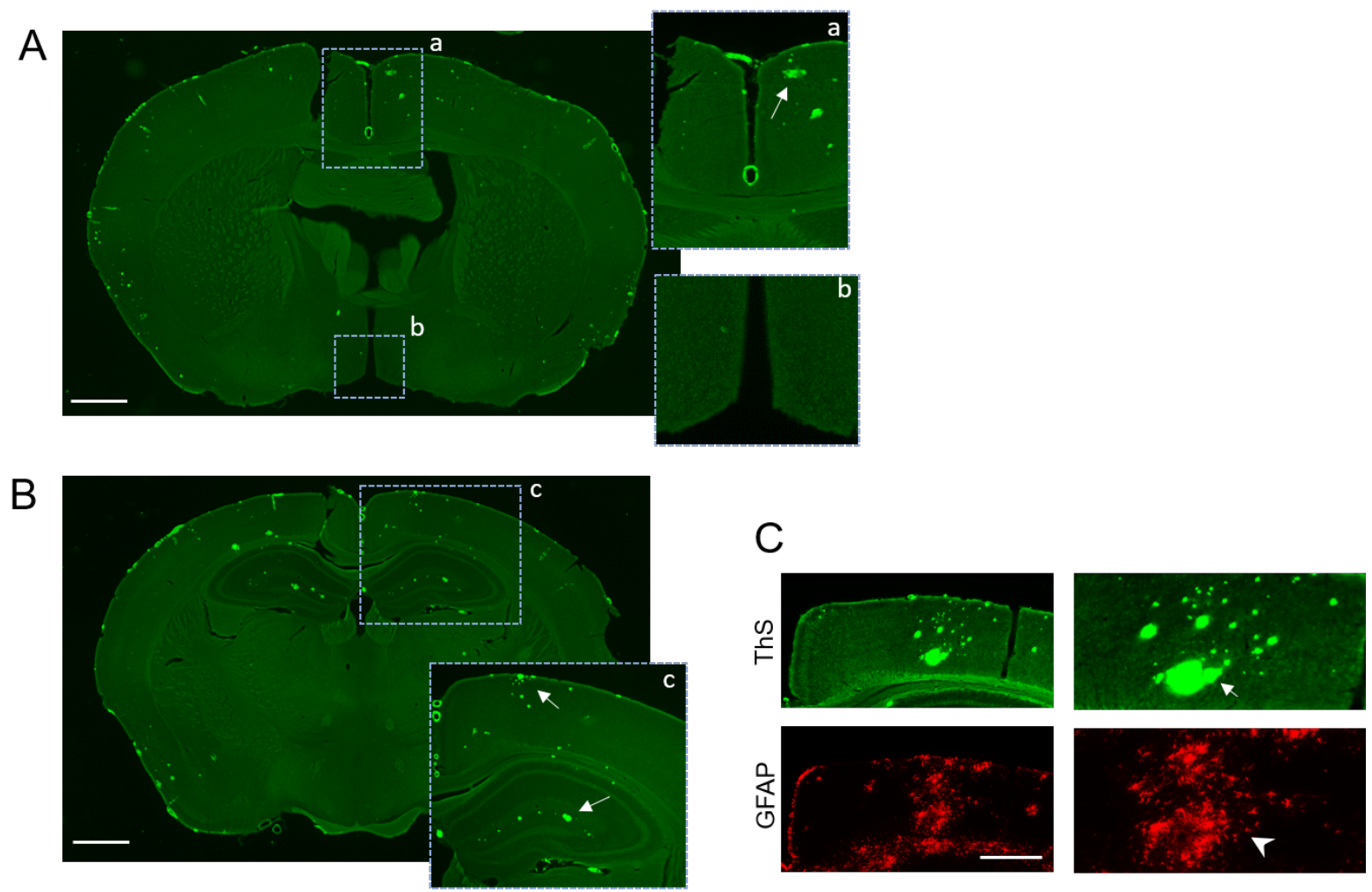

Supplementary Fig. 1. Representative histological images of thioflavin-S (ThS) staining in the brain slices containing (A) cingulate cortex and SCN regions and (B) parietal cortex and hippocampus regions. Amyloid $(\mathrm{A} \beta)$ plaques were visible in various cortex and hippocampus regions, however no $\mathrm{A} \beta$ plaques were observed in SCN region.; (C) Double staining of ThS and GFAP showing A $\beta$ plaques (arrow) surrounded by reactive astrocytes (arrow head). Scale bar: $500 \mu \mathrm{m}$ (in A and B) and $250 \mu \mathrm{m}$ (in C). 